\title{
Covid-19-Maßnahmen im Strafrecht - Ein Rückblick nach eineinhalb Jahren Pandemie
}

\section{Alois Birklbauer}

Nachdem zu Beginn der COVID-19-Pandemie auch im Bereich des Strafrechts vorwiegend die Frage im Zentrum der Diskussion stand, inwieweit die getroffenen Maßnahmen mit Blick auf die Grund- und Freiheitsrechte verhältnismäßig waren, soll nach mehr als einem Jahr der Fokus darauf gerichtet werden, wie die vorhandenen bzw. adaptierten Straf-, Verfahrens- und Vollzugsnormen von der Praxis genutzt wurden. Nach einer Einleitung (1.) werden in einem ersten Teil der strafrechtliche Umgang mit der Gefährdung anderer durch positiv getestete Personen untersucht (2.) sowie die mögliche Strafbarkeit von „Impf-Vordrängern“ (3.) und „Test-Fälschern“ (4.). In einem zweiten Teil sollen aus dem Bereich des Strafverfahrensrechts die Themen Videovernehmung und Begrenzung der Öffentlichkeit bei Verhandlungen angesprochen werden (5.), zumal diese immer noch aktuell sind und auch darüber diskutiert wird, diese zumindest teilweise pandemieunabhängig fortzuführen. Aus dem Strafvollzugsbereich sollen die Themen Vollzugsaufschub und Besuchsbeschränkungen (6.) angesprochen werden. Zusammenfassende Schlussfolgerungen stehen am Ende des Beitrags (7.).

\section{Einleitung}

Die COVID-19-Pandemie hat den Umgang der Menschen miteinander wesentlich verändert und zu unterschiedlichen Forderungen an die Politik geführt. Während viele Unterstützung im Schutz vor der Erkrankung gefordert und die staatlich angeordneten freiheitsbeschränkenden Maßnahmen goutiert haben, beriefen sich deutlich weniger auf ihre Freiheiten, wenngleich sich deren Anteil im Lauf der Pandemie erhöht hat. Die Regierung hat sich umgehend auf die Seite der Mehrheit gestellt und zum Teil mit Angstmache um Zustimmung für die verordneten Einschränkungen geworben. Mit zunehmender Dauer der Pandemie ist deren Akzeptanz geschwunden, was bisweilen mit der „Pandemiemüdigkeit der Bevölkerung“ umschrieben wurde. Doch die Kritik verstärkte sich nicht zuletzt auch 
deswegen, weil das Pandemie-Management immer wieder Fehler gemacht hat und diese mehr und mehr bekannt wurden.

Die letztlich rasche Verfügbarkeit wirksamer Impfstoffe erlaubte es, eine Rücknahme der Beschränkungen anzudenken. Dabei war es mit Blick auf die Grundrechte naheliegend, bei Personen mit einem geringen epidemiologischen Risiko, weil sie etwa bereits geimpft oder genesen sind, die Grundrechtsbeschränkungen zurückzunehmen. Da es jedoch infolge der (anfangs) beschränkten Impfstofflieferungen eine Weile gedauert hat, bis der breiten Bevölkerung ein Impfangebot gemacht werden konnte, und seitens der Politik eine Reihung nach Schutzbedürftigkeit von Bevölkerungsgruppen und Systemrelevanz bestimmter Berufstätigkeiten vorgenommen wurde, war bald klar, dass manche ihre unbegrenzte Freiheit rascher erlangen werden (können) als andere. Insofern war es naheliegend, dass immer wieder Personen versuchten, sich bei der Impfung vorzudrängen, um die Freiheitsbeschränkungen loszuwerden. Mit niederschwelligen Testmöglichkeiten und der gleichwertigen Behandlung mit Geimpften und Genesenen (3-G-Regel) wurde letztlich versucht, Überlegungen der Gerechtigkeit zu entsprechen. ${ }^{1}$ Freilich war die Debatte damit nicht beseitigt, zumal die vorgegebene Impfreihung vielfach nur schwer nachvollziehbar war. Erinnert sei etwa an den Plan in manchen Bundesländern, die Mitglieder der Freiwilligen Feuerwehren infolge deren Systemrelevanz vor dem Kindergartenpersonal zu impfen, das täglich durch die fehlende Testung der Kinder und den unvermeidbaren engen Kontakt mit diesen einem viel größeren Infektionsrisiko ausgesetzt war. In der Debatte um die Impfstoffverteilung zeigte sich wieder einmal, dass die geringere Wertschätzung gegenüber bestimmten Berufsgruppen bzw. Angehörigen einer Gesellschaft die Entscheidung über die Priorisierung und die Grundrechtsbeschränkungen mitprägte. So blieben etwa die Besuchsbeschränkungen in Alten- und Pflegeheimen trotz weitgehender Durchimpfung noch lange aufrecht oder auch die so genannte „Bildungsampel“ in Kindergärten trotz Entspannung der Situation bis Juni 2021 auf „Rot“, was mit wesentlichen (und nicht mehr erforderlichen) Einschränkungen für Personal und Kinder verbunden war.

In der folgenden Darstellung zum Umgang mit der Pandemie im Bereich des Strafrechts soll es nicht nur um die Anwendung bestimmter

1 Siehe dazu etwa die Stellungnahme der Bioethikkommission beim Bundeskanzleramt (BEK) zum Thema Rechtliche und ethische Fragen im Zusammenhang mit geimpften und genesenen Personen in der COVID-19-Pandemie, in: https://www. bundeskanzleramt.gv.at/themen/bioethikkommission/publikationen-bioethik.html [3.7.2021]. 
Strafnormen durch die Gerichtsbarkeit gehen, sondern vor allem auch um den Umgang mit den einer Straftat Beschuldigten sowie den wegen einer Straftat Verurteilten. Da auch diese Gruppen letztlich keine Lobby haben, ist es nicht verwunderlich, wenn sie zu jenen gehören, denen durch die Pandemie lange Zeit über Gebühr hohe (zusätzliche) Freiheitsbeschränkungen auferlegt wurden.

\section{Die strafbare Gefährdung anderer durch positiv auf COVID-19 getestete Personen}

Das materielle Strafrecht blieb im Rahmen der COVID-19-Krise von Neuerungen verschont. Vorherrschend war die Ansicht, dass bereits ausreichende Strafnormen vorhanden sind und es daher nicht nötig sei nachzuschärfen. So schützen die Bestimmungen des materiellen Strafrechts sowohl das Individualrechtsgut Leib und Leben ( $\$ \$ 75 \mathrm{ff}$ Strafgesetzbuch $[\mathrm{StGB}]^{2}$ ), als auch das Universalrechtsgut Leben und Gesundheit der Allgemeinheit („Volksgesundheit") vor Gefährdung von Menschen durch übertragbare Krankheiten $(\mathbb{S} S 178 \mathrm{f} \mathrm{StGB}) .^{3}$ Kann über das Individualrechtsgut Gesundheit vom jeweiligen Rechtsgutsträger dahingehend verfügt werden, dass der Verursacher einer Gesundheitsschädigung Straffreiheit erlangt, wenn der Geschädigte sich bewusst der Gesundheitsgefährdung ausgesetzt hat, ist das Universalrechtsgut „Volksgesundheit“ einer strafbefreienden Disposition seitens des Geschädigten von vornherein entzogen.

\subsection{Körperverletzungsdelikte}

Die Körperverletzungsdelikte ( $\$ \$ \$ 83 \mathrm{ff}$ StGB) erfassen neben der Verletzung eines konkreten Individuums in seiner körperlichen Integrität (Unversehrtheit) gleichermaßen die Gesundheitsschädigung als Beeinträchtigung des physischen und/oder psychischen Wohlbefindens, welches Krankheitswert im medizinischen Sinn besitzt. ${ }^{4}$ Dabei wird die Erheblichkeitsschwelle niedrig angesetzt, reicht doch z. B. für eine Körperverletzung $(\mathbb{S} 83$ Abs. 1 StGB) bereits eine nicht allzu große Hautrötung aus, um den Tatbe-

2 Bundesgesetz vom 23. Jänner 1974 über die mit gerichtlicher Strafe bedrohten Handlungen (Strafgesetzbuch - StGB), BGBl. 60/1974.

3 Vgl. zu diesem Rechtsgut etwa Murschetz in WK $\mathrm{W}^{2} \mathrm{StGB} \$ \mathbb{S} 178,179 \mathrm{Rz} 1$.

4 Vgl. dazu etwa Burgstaller/Fabrizy in $\mathrm{WK}^{2} \mathrm{StGB} \ 83 \mathrm{Rz} 9 \mathrm{ff}$. 
stand zu begründen. ${ }^{5}$ Mit Blick auf diese niedrige Schwelle wurde zu Beginn der Pandemie davon ausgegangen, dass eine COVID-19-Infektion, unabhängig von ihrem konkreten Verlauf, stets die Tatbestandsschwelle für eine Gesundheitsschädigung überschreitet und bei mehr als 24-tägiger Dauer sogar eine „schwere Körperverletzung“ ( $\$ 84$ Abs. 1 StGB) vorliegt. ${ }^{6}$ Die Frage, ob eine positiv getestete Person auch tatsächlich krank im medizinischen Sinne ist, wurde nicht weiter thematisiert, sondern es wurde dem medizinischen Mainstream gefolgt, der letztlich die positive Testung als infektiös und damit den Getesteten als erkrankt betrachtete.

Inzwischen musste sich die Judikatur zwar nicht mit dem Thema COVID-19-Infektion als Krankheit, aber sehr wohl mit der Frage Infektion und der daraus resultierenden Ansteckungsgefahr auseinandersetzen. So hat z. B. das Oberlandesgericht (OLG) Linz in einem Leitsatz festgehalten, dass die Frage der Ansteckungsgefahr letztlich aufgrund der Virenlast anhand des Laborbefundes zu klären ist. ${ }^{7}$ Diese differenzierte Sichtweise muss auch für den Begriff der Gesundheitsschädigung als Unterfall der Körperverletzung dazu führen, dass trotz der grundsätzlich niedrigen Schwelle für dieses Tatbestandsmerkmal das Vorliegen von Krankheitssymptomen entscheidend sein muss, um von einer „Körperverletzung“ im Sinne der strafrechtlichen Terminologie sprechen zu können. Fehlt es infolge eines symptomlosen Verlaufs der Infektion an einer Beeinträchtigung des physischen und/oder psychischen Wohlbefindens des Infizierten, liegt keine Gesundheitsschädigung vor und scheitert eine Subsumtion unter die Körperverletzungsdelikte nach $\$ \mathbb{S} 83 \mathrm{ff} S \mathrm{tGB}$. Das gilt sowohl für den vorsätzlichen als auch den fahrlässigen Bereich. ${ }^{8}$

5 Vgl. OGH 11 Os 180/76 = SSt 48/20: linsengroßer Hautdefekt am Grundgelenk des 5. Fingers links und oberflächliche Hautabschürfungen an der linken Augenbraue.

6 Vgl. etwa mit Nachweisen Birklbauer in Resch, Corona-HB Kap 16 Rz 2 ff.

7 OLG Linz 7 Bs 48/21i vom 22.4.2021 = RIS-Justiz RL0000214; siehe dazu näher unten im Text bei FN 19.

8 Auf eine allfällige Versuchsstrafbarkeit, wenn der*die Täter*in davon ausgeht, infiziert und ansteckend zu sein, sei hier nicht eingegangen, weil die Frage der absoluten Untauglichkeit des Versuchs, die eine Straffreiheit bewirken würde (vgl. $\mathbb{\$} 15$ Abs. 3 StGB) einer umfassenden Darstellung bedürfen würde, die den Rahmen sprengen würde. 


\subsection{Gefährdung der „Volksgesundheit“}

Die Gefährdung von Menschen durch übertragbare Krankheiten $(\mathbb{S} \mathbb{S} 178 \mathrm{f}$ StGB) setzt zunächst eine geeignete Handlung des Infizierten voraus, um die Gefahr der Verbreitung einer übertragbaren Krankheit unter Menschen herbeizuführen. Dabei gelten als übertragbare Krankheiten jene, bei denen ein Krankheitserreger unmittelbar oder mittelbar von einem Individuum auf ein anderes übergehen kann. ${ }^{9}$ Im Unterschied zu den Körperverletzungsdelikten kommt es hier nicht darauf an, dass tatsächlich jemand durch das Verhalten eines anderen infiziert wird, sondern es reicht aus, dass die Gefabr einer Verbreitung, also eine Zunahme des Ausdehnungsbereichs der Krankheit herbeigeführt wird, ohne dass es epidemischer Ausmaße bedürfe. ${ }^{10}$

Damit die Strafbarkeit dieses Delikts nicht zu sehr ausufert, muss die Krankheit ihrer Art nach zu den zumindest beschränkt anzeige- oder meldepflichtigen Krankheiten gehören, um nicht auch relativ ungefährliche Krankheiten wie Schnupfen oder Grippe unter das Delikt zu subsumieren. ${ }^{11}$ Diese Anzeige- bzw. Meldepflicht ist eine objektive Bedingung der Strafbarkeit, ${ }^{12}$ die dem Täter weder bewusst noch für ihn erkennbar gewesen sein muss.

COVID-19 ist eine übertragbare Krankheit, deren Erreger unmittelbar oder mittelbar von einem Individuum auf ein anderes übergehen kann. Die Krankheit ist meldepflichtig nach $₫ 1$ Abs. 1 Z. 1 Epidemiegesetz, ${ }^{13}$ wodurch auch die genannte objektive Bedingung der Strafbarkeit als Voraussetzung für eine Subsumtion unter $\$ \$ 178 \mathrm{f}$ StGB erfüllt ist.

Da die $\mathbb{S} 178 \mathrm{f}$ StGB als abstrakte (potentielle) Gefährdungsdelikte ausgestaltet sind, setzen sie voraus, dass mit dem gesetzten Verhalten die Gefahr einhergeht, die Verbreitung einer ansteckenden Krankheit unter Menschen

9 Vgl. Leukauf et al., StGB ${ }^{4} \$ 178 \mathrm{Rz} 2$.

10 Vgl. Leukauf et al., StGB 4 \$ $178 \mathrm{Rz} 8$; Murschetz in WK ${ }^{2}$ StGB $\$ \mathbb{S} 178,179 \mathrm{Rz} 2$.

11 Vgl. Leukauf et al., StGB $4 \$ \$ 178 \mathrm{Rz} 5$.

12 Vgl. Leukauf et al., StGB 4 \$ 178 Rz 4; Murschetz in WK ${ }^{2}$ StGB $\$ \$ 178,179$ Rz 5.

13 Epidemiegesetz 1950, BGBl.186/1950 (EpidemieG). Die Bestimmung nennt MERS-CoV (Middle East Respiratory Syndrome Coronavirus/,neues CoronaVirus“). Verschiedentlich wird darauf hingewiesen, dass es sich bei SARS-CoV-2 nicht um das genannte „neue Coronavirus“ handelt, sondern um das „2019 neuartiges Coronavirus“ (2019-nCoV). Daher beruhe die Meldepflicht auf der nach $\$ 1$ Abs 2 EpidemieG 1950 ergangenen Verordnung des Bundesministeriums für Arbeit, Soziales, Gesundheit und Konsumentenschutz (BMASGK) über anzeigepflichtige übertragbare Krankheiten 2020 (BGBl II 2020/15); vgl. dazu mit Nachweisen Birklbauer in Resch, Corona-HB Kap 16 Rz 20. 
herbeizufübren. Dies wurde zu Beginn der Pandemie relativ rasch für jene angenommen, die mit dem COVID-19-Virus infiziert waren und deshalb einen Absonderungsbescheid von der Gesundheitsbehörde erhalten hatten. So wurde z. B. eine infizierte Frau wegen vorsätzlicher Gefährdung von Menschen durch übertragbare Krankheiten nach $₫ 178$ StGB zu einer Geldstrafe verurteilt, weil sie (mit Mundschutz) eine kleine Runde spazieren gegangen war, obwohl sie die Runde so gewählt hatte, dass sie niemandem begegnet ist. ${ }^{14}$ Ein 58 -jähriger Mann, der positiv getestet war und letztlich aus Angst, seinen Job zu verlieren, arbeiten ging, zumal er sich nicht krank fühlte, wurde wegen des genannten Delikts gar zu einer (bedingten) Freiheitsstrafe von sechs Monaten und einer unbedingten Geldstrafe verurteilt. ${ }^{15}$ Eine ähnlich hohe Strafe wurde gegen eine Frau verhängt, die trotz COVID-19-Infektion an einem Postschalter Geld an ihre kranke Enkelin in Bosnien überweisen wollte, nachdem der Überweisungsversuch durch eine Kollegin gescheitert war. ${ }^{16}$ Wegen fahrlässiger Gefährdung nach $\mathbb{1} 179$ StGB stand eine Studentin vor Gericht, die bei einem Arztbesuch nicht mitgeteilt hatte, dass ihr Lebensgefährte als „Kontaktperson 1" in Quarantäne ist, weil dessen Eltern positiv getestet wurden. Das Verfahren wurde in diesem Fall schließlich diversionell eingestellt, nachdem die Studentin sich bereit erklärt hatte, 40 Stunden gemeinnützige Leistungen zu erbringen. ${ }^{17}$ Auch das Landesverwaltungsgericht Wien hat in einem Erkenntnis undifferenziert festgestellt, dass die Nichteinhaltung eines Absonderungsbescheids zwecks Verhütung der Weiterverbreitung von COVID-19 das Tatbild der Gefährdung von Menschen durch übertragbare Krankheiten gemäß $\$ \$ 178$ und 179 StGB erfüllt.. ${ }^{18}$

14 Vgl. Die Presse: 1200 Euro Strafe für Spaziergang mit Covid-19 (25.7.2020), in: https://www.diepresse.com/5844184/1200-euro-strafe-fur-spaziergang-mit-covid-19 [3.7.2021].

15 Vgl. ORF Wien: CoV-positiver Mann ging arbeiten: Verurteilt (17.8.2020), in: https://wien.orf.at/stories/3062649/ [3.7.2021].

16 Vgl. Der Standard: Das Coronavirus im Strafrecht: Erstes Urteil wegen verletzter Quarantäne (22.7.2020), in: https://www.derstandard.at/story/2000118846653/das -coronavirus-im-strafrecht-erstes-urteil-wegen-verletzter-quarantaene [3.7.2021].

17 Widmayer: „Personen fahrlässig gefährdet": Diversion für Studentin in Salzburger Covid-Strafprozess, in: Salzburger Nachrichten (2.2.2021), https:/www.sn.at/s alzburg/chronik/personen-fahrlaessig-gefaehrdet-diversion-fuer-studentin-in-salzb urger-covid-strafprozess-99244438 [3.7.2021].

18 Vgl. LVwG Wien VGW-001/016/8713/2020 vom 23.7.2020. Dies war jedoch insoweit eine Formalentscheidung, als mit dieser Begründung die Zuständigkeit für verwaltungsstrafrechtliche Maßnahmen verneint wurde. Als Entscheidung in der Sache kann dies nur bedingt gewertet werden. 
Inzwischen ist die Rechtsprechung differenzierter. So hat etwa das OLG Linz in seiner bereits angesprochenen Entscheidung vom 22.4.2021 ${ }^{19}$ festgestellt, dass nicht jede COVID-19-Infektion einer Person mit einer potentiellen Ansteckungsgefahr für andere Personen einhergeht und daher die Frage der Ansteckungsgefahr letztlich aufgrund der Virenlast anhand des Laborbefundes zu klären sei. Der Fall betraf einen Mann, der acht Tage nach positiver Testung auf COVID-19 und zwei Tage vor Ende des Absonderungsbescheids zur Behörde ging, um eine Meldebescheinigung zu beantragen. Das OLG Linz hat hierzu festgehalten, dass einem Absonderungsbescheid hinsichtlich der Gefahr für die Gesundheit anderer zwar eine Indizfunktion zukomme, die Ansteckungsgefahr, die von einem Infizierten ausgeht, aber entscheidend sei. Da aus der 4. COVID-19 SchuMaV20 hervorgehe, dass bei einer positiv auf COVID-19 getesteten Person mit einem CT-Wert größer als 30 keine Ansteckungsgefahr mehr bestehe, ${ }^{21}$ ist daraus zu folgern, dass nicht jede COVID-19-Infektion einer Person mit einer potenziellen Ansteckungsgefahr für andere Personen einhergehe und die Frage der Ansteckungsgefahr daher letztlich aufgrund der Virenlast anhand des Laborbefundes zu klären sei.

In ähnlicher Denkweise hat auch das OLG Graz festgehalten, dass das Husten einer nicht infizierten Person (Viruslast: null) nicht geeignet sei, die Gefahr der Verbreitung der übertragbaren Krankheit COVID-19 unter Menschen herbeizuführen, und daher auch nicht als Tathandlung des $\$ 178$ StGB in Betracht komme. ${ }^{22}$ In diesem Fall hat die angeklagte Frau sich einer Amtshandlung widersetzt, die maximal einen Meter entfernt stehenden Beamten angehustet und geäußert, wenige Tage davor in einem Ort gewesen zu sein, der „wegen Corona“ unter Quarantäne stehe. Wenige Stunden nach dem Vorfall wurde sie negativ auf COVID-19 getestet. Zufolge der nicht vorliegenden Infizierung der Angeklagten mit dem Krankheitserreger SARS-CoV-2 wurde durch ihr Verhalten nicht einmal eine bloß theoretische Möglichkeit der Rechtsgutsbeeinträchtigung in Form der Verbreitung der Krankheit COVID-19 geschaffen. Die Handlung war

19 Vgl. OLG Linz 7 Bs 48/21i vom 22.4.2021 = RIS-Justiz RL0000214; zu den Auswirkungen dieser Entscheidung für die Körperverletzungsdelikte siehe oben im Text bei FN 7.

20 4. COVID-19-Schutzmaßnahmenverordnung (4. COVID-19-SchuMaV), BGB1. II 2021/58.

21 Das Judikat verweist auf $₫ 10$ Abs $4 \mathrm{Z} 2$, Abs 4 a Z 2 und $₫ 11$ Abs 4 Z 2 der 4. COVID-19-SchuMaV.

22 OLG Graz 1 Bs 10/21m vom 5.3.2021 = RIS-Justiz RG0000192. 
somit abstrakt nicht geeignet, die Gefahr der Verbreitung einer Krankheit herbeizufübren, wodurch eine Verwirklichung der $\$ \mathbb{S} 178 \mathrm{f} \mathrm{StGB}$ ausscheide.

Damit lässt sich zur Gefährdung anderer mit ansteckenden Krankheiten ( $\mathbb{S} 178 \mathrm{f} \mathrm{StGB}$ ) zusammenfassend festhalten, dass die Tatsache einer positiven Testung auf COVID-19 oder auch die Infektion mit dem Virus nicht ausreichen, um die genannten Delikte zu verwirklichen. Es muss überdies von den Strafverfolgungsbehörden nachgewiesen werden, dass von einer konkreten Person eine Ansteckungsgefahr ausgegangen ist, indem - nach derzeitigem Wissensstand - im Handlungszeitpunkt ein CT-Wert $>30$ vorgelegen ist. Die ursprünglich in der Literatur vertretenen Positionen, die eine positive Testung oder auch eine Infektion mit COVID-19 als ausreichend erachtet haben, um die genannten Gefährdungsdelikte zu verwirklichen, sind damit überholt.

\section{Das Vordrängen bei der Impfung als strafbares Verhalten}

Impfungen gegen COVID-19 waren, wie bereits in der Einleitung erwähnt, zu Beginn des Jahres 2021 heiß begeht, infolge der mit dem Impfplan vorgegebenen Priorisierung ${ }^{23}$ aber nicht allgemein erhältlich. Durch die mit der Impfmöglichkeit verbundene Euphorie war es naheliegend, dass Personen, die noch nicht an der Reihe waren, sich um eine Impfung drängten. So wurde etwa von Bürgermeistern berichtet, die prioritär geimpft wurden, weil die Gemeinde Betreiberin des Seniorenzentrums war. ${ }^{24} \mathrm{Be}-$ sonders aufsehenerregend war ein Fall in einer oberösterreichischen Gemeinde, in dem von 44 für ein Pflegeheim bestimmten Impfdosen nur 16 an Heimbewohner*innen und -mitarbeiter*innen verimpft wurden. Der Rest ging an heimfremde Personen. ${ }^{25}$ Auch gab es Berichte über eine Vorreihung nach einer Spende für das Pflegeheim. ${ }^{26}$ Die strafrechtliche Erfassung solcher Sachverhalte gestaltet sich schwierig.

23 Dazu wurde vom Bundesministerium für Soziales, Gesundheit, Pflege und Konsumentenschutz ein COVID-19 Impfplan verfasst, in der aktuellen Fassung abrufbar unter https:/www.sozialministerium.at/Corona-Schutzimpfung/Corona-Schu tzimpfung---Durchfuehrung-und-Organisation.html [3.7.2021].

24 Vgl. ORF-Oberösterreich: Impfung: Weitere Politiker-Vorreihungen in OÖ (19.1.2021), in: (https:/ooe.orf.at/stories/3085673/ [3.7.2021].

25 Vgl. ORF-Oberösterreich: Politiker wurden in Pflegeheim geimpft (17.1.2021), in: https://ooe.orf.at/stories/3085387/ [3.7.2021].

26 Vgl. Pessl: Wird man mit einer Spende für die Coronaimpfung vorgereiht?, in: Salzburger Nachrichten (19.1.2021), https:/www.sn.at/panorama/oesterreich/wir d-man-mit-einer-spende-fuer-die-coronaimpfung-vorgereiht-98542825 [3.7.2021]. 


\subsection{Vorreihung als Gegenleistung}

Am einfachsten ist die Vorreibung nach einer Spende als gleichsame Gegenleistung zu beurteilen, weil hier die Korruptionsdelikte ( $\mathbb{S} 304 \mathrm{ff}$ StGB) anwendbar sein könnten. Diese erfassen auch den staatsnahen Bereich, indem sie Amtsträger betreffen ( $\$ 74$ Abs. 1 Z. 4a StGB) und darunter alle Organe und Bediensteten eines Unternehmens fallen, an denen Gebietskörperschaften wie Bund, Länder oder Gemeinden mit mindestens 50 Prozent beteiligt sind oder dessen Unternehmensgebarung vom Rechnungshof des Bundes oder der Länder kontrolliert wird. Nach dieser Begriffsbestimmung zählen etwa die Organe von Sozialhilfeverbänden oder die Bediensteten von Heimen, die von diesen betrieben werden, zu den Amtsträgern.

Bei den Tatbeständen der Korruptionsdelikte gibt es sowohl solche für die Nehmerseite ( $\$ \$ 304-306$ StGB) als auch die Geberseite $(\mathbb{S} 307-307 \mathrm{~b}$ StGB). Zur Verwirklichung muss auf Geberseite ein Vorteil zumindest versprochen werden, auf Nehmerseite reicht es, sich einen Vorteil versprechen zu lassen. Unter einem Vorteil ist jede Leistung materieller und immaterieller Art zu verstehen, die den Täter besser stellt (die nützlich ist) und auf die er keinen rechtlich begründeten Anspruch hat. ${ }^{27}$ Nach dem Gesetzeswortlaut ist es nicht erforderlich, dass der Vorteil an den fließt, der ein Amtsgeschäft vornimmt, sondern das Delikt ist auch dann verwirklicht, wenn der Begünstigte ein Dritter ist, etwa das Unternehmen, für das der Amtsträger tätig ist. ${ }^{28}$ Nun fällt das Impfen zwar nicht in die Hoheitsverwaltung, sondern es wird von Ärzt"innen im Rahmen der Privatwirtschaftsverwaltung durchgeführt. Dies bedeutet für die Korruptionsdelikte, dass es dann am Vorteil fehlt, wenn der Zuwendung an den Amtsträger ein zivilrechtlich gültiger entgeltlicher Vertrag zu Grunde liegt. ${ }^{29}$ Verträge, die eine Impfvorreihung als Gegenleistung beinhalteten, sind aber wohl als sittenwidrig (vgl. $\$ 879 \mathrm{ABGB}^{30}$ ) einzustufen, weshalb es an deren zivilrechtlicher Gültigkeit fehlt. Die Verwirklichung der Korruptionsdelikte durch die geschilderten Verhaltensweisen scheint somit evident zu sein. Medienberichte über Verurteilungen in Fällen von Impfvorreihungen als Gegenleistung existieren nicht, vielleicht auch, weil sich die erhobenen Vorwürfe letztlich doch in Luft aufgelöst haben.

27 Vgl. dazu mit Nachweisen zum Meinungsstand Birklbauer in Resch/Wallner, Handbuch Medizinrecht ${ }^{3}$ Kap X Rz 186 ff.

28 Vgl. OGH 17 Os 8/16d vom 6.6.2016 = RIS-Justiz RS0130816.

29 Vgl. dazu OGH 17 Os 8/16d vom 6.6.2016 = RIS-Justiz RS0130815.

30 Allgemeines bürgerliches Gesetzbuch für die gesammten deutschen Erbländer der Oesterreichischen Monarchie; JGS Nr. 1811/946 in der geltenden Fassung. 


\subsection{Vorreibung obne Gegenleistung}

Die Vorreihung ohne Gegenleistung, etwa aus Freundschaft, Sorge um die Familie oder auch infolge politischer Einflussnahme, lässt sich strafrechtlich nicht fassen. Das Delikt des Missbrauchs der Amtsgewalt ( $\$ 302 \mathrm{StGB}$ ), an das primär zu denken ist, scheitert daran, dass das Impfen keinen Akt der Hoheitsverwaltung darstellt und ein solcher Voraussetzung für den Amtsmissbrauch ist (Amtsgeschäfte „in Vollziehung der Gesetze“). Hoheitsverwaltung liegt dann vor, wenn der Staat zur Erreichung seiner Ziele die ihm auf Grund seiner spezifischen Macht gegebene einseitige Anordnungsbefugnis gebraucht, demnach als Träger dieser besonderen Befehls- und Zwangsgewalt auftritt, insbesondere durch Einsatz bestimmter Rechtsformen wie Verordnung, Bescheid oder Akt unmittelbarer verwaltungsbehördlicher Befehls- und Zwangsgewalt. ${ }^{31}$ Wenn Unregelmäßigkeiten bei der Durchführung von Vorsorgeuntersuchungen im Bereich des MutterKind-Passes durch Ärzt*innen im Bereich öffentlicher Gesundheitsversorgung keinen Amtsmissbrauch begründen können, weil sie nicht Teil der Hoheitsverwaltung sind, ${ }^{32}$ muss dies ebenso für COVID-19-Impfungen gelten. Auch andere Delikte sind für die Vorreihung ohne Vorteile nicht ersichtlich, weshalb es auch naheliegend war, dass die infolge der Medienberichte eingeleiteten strafrechtlichen Ermittlungen zu einer Verfahrenseinstellung geführt haben. ${ }^{33}$ Debatten über die Einführung eines eigenen Straftatbestands für Impf-Vordrängler wurden in Österreich letztlich nicht wirklich geführt, zumal relativ bald klar war, dass es in absehbarer Zeit ohnehin ausreichend Impfstoff für alle Impfwilligen geben werde. Insofern blieb es allein bei der moralischen Verantwortlichkeit.

\section{Die Strafbarkeit gefälschter „Eintritts-Tests“}

Mit der Lockerung der COVID-19-Maßnahmen wurden für alle, die am sozialen Leben wieder teilnehmen wollten, aber (noch) nicht geimpft und auch nicht genesen waren, die so genannten ,Eintritts-Tests' von erheblicher Bedeutung. Mit einem solchen konnten zunächst körpernahe Dienst-

31 Vgl. OGH 17 Os 34/15a vom 6.6.2016 = RIS-Justiz RS0130809.

32 So explizit OGH 17 Os 25/14a vom 11.8.2014 = RIS-Justiz RS0118428 (T6).

33 Vgl. etwa Oberösterreichische Nachrichten: Impfungen in Innviertler Pflegeheimen: Staatsanwaltschaft stellte Ermittlungen ein (30.3.2021), in: https:/www. nachrichten.at/oberoesterreich/impfungen-in-innviertler-pflegeheimen-staatsa nwaltschaft-stellte-ermittlungen-ein;art4,3376075 [3.7.2021]. 
leistungen, später auch Restaurantbesuche, Hotelaufenthalte, Kulturveranstaltungen usw. erlangt werden. Da das Testen mit Aufwand verbunden und auch nicht immer angenehm war, stieg die Versuchung, einen fremden Test vorzuweisen oder Testdaten wie den Namen des Getesteten oder das Testdatum zu verändern, um dadurch in den Genuss der angestrebten Lockerung zu kommen. Da das entsprechende Dokument des Gesundheitsministeriums, welches ein negatives Testergebnis bestätigte und elektronisch zugesendet wurde, anfangs - mit Ausnahme des Briefkopfs nicht schreibgeschützt war, ${ }^{34}$ war es auch technisch nicht allzu schwierig, derartige Manipulationen vorzunehmen.

\subsection{Urkundenfälschung}

Die strafrechtliche Relevanz der genannten Verhaltensweisen war deutlich höher als beim Impf-Vordrängen ${ }^{35}$ und wird sich in vergleichbarer Weise auch für Manipulationen beim so genannten „Grünen Pass“ stellen. Als Delikt kommt hier Urkundenfälschung ( $\$ 223$ StGB) in Betracht, welches sowohl das Fälschen oder Verfälschen einer Urkunde erfasst (Abs. 1) als auch den Gebrauch einer solchen Urkunde (Abs. 2). Entscheidend ist stets, dass die Tathandlung gesetzt wird mit dem Vorsatz, die falsche oder verfälschte Urkunde im Rechtsverkehr zum Beweis eines Rechtes, eines Rechtsverhältnisses oder einer Tatsache zu verwenden. Bloße Fälschungen zum Spaß, etwa als Geburtstagsgag, reichen nicht zur Tatbestandserfüllung.

Eine Urkunde ist entsprechend der Legaldefinition in $\$ 74$ Abs. 1 Z. 7 StGB eine Schrift, die errichtet worden ist, um ein Recht oder ein Rechtsverhältnis zu begründen, abzuändern oder aufzuheben oder eine Tatsache von rechtlicher Bedeutung zu beweisen. Ein bestätigtes negatives Testergebnis ist unter diesen Urkundenbegriff zu subsumieren, zumal darin eine Tatsache von rechtserheblicher Bedeutung bestätigt wird, weil sie Voraussetzung für die Annahme bestimmter Leistungen ist.

Das Tatbestandsmerkmal ,falsch“ bei der Urkundenfälschung nach $\$ 223$ StGB bezieht sich nicht auf den Inhalt der Urkunde, sondern allein auf den Umstand, dass dem Urkundenaussteller etwas unterstellt wird, das

34 Vgl. Laglstorfer: Sicherheitslücke bei offiziellen Covid-Testergebnissen, in: Oberösterreichische Nachrichten (6.2.2021), https:/www.nachrichten.at/oberoesterrei ch/sicherheitsluecke-bei-offiziellen-covid-testergebnissen;art4,3347472 [3.7.2021]; nachdem das Bundesministerium auf diesen Umstand aufmerksam (gemacht) wurde, gab es Nachbesserungen.

35 Vgl. dazu Neuhofer, JSt 2021, S. 231. 
er so nicht bestätigt hat. Insofern wird falsch nicht als unrichtig, sondern als unecht verstanden. Wer ein Blankoformular einer Behörde mit seinen Daten ausfüllt, stellt eine falsche Urkunde her, weil er der Behörde eine Aussage unterstellt, die sie so nicht gemacht hat. Wird ein von der Behörde ausgestelltes Formular verändert, verfälscht oder anderweitig nachträglich verändert, wird damit eine Urkunde verfälscht. Insofern lassen sich die geschilderten Manipulationen von bestätigten Testergebnissen einfach unter die Strafnorm des $\mathbb{2 2 3}$ StGB subsumieren. Lediglich das Verwenden einer für eine andere Person ausgestellten Bestätigung, die aber nicht verändert wird, begründet keine strafrechtliche Relevanz, weil hier nichts am Dokument verfälscht wird. Das Delikt des Gebrauchs fremder Ausweise ( $\$ 231$ StGB) scheitert am Ausweischarakter der Testbestätigung.

\subsection{Datenfälschung}

Eine Urkunde im Sinne von $₫ 74$ Abs. 1 Z. 7 StGB wird erst hergestellt, wenn sie als Schriftstück vorliegt, also z. B. die am elektronischen Dokument vorgenommene Änderung ausgedruckt wird. Wird ein elektronisch übermitteltes Dokument lediglich am Computer oder Smartphone verändert ohne dass ein Ausdruck erfolgt, scheiden die Urkundendelikte mangels Urkunde aus. Freilich bedeutet dies keineswegs eine Straffreiheit des gesetzten Verhaltens, sondern für solche Fälle kommt eine Datenfälschung nach $\$ 225$ a StGB in Betracht. Dieses Delikt verwirklicht unter anderem, wer durch Eingabe oder Veränderung falsche Daten herstellt oder echte Daten verfälscht, wiederum mit dem Vorsatz, dass die Daten im Rechtsverkehr zum Beweis eines Rechtes, eines Rechtsverhältnisses oder einer Tatsache gebraucht werden.

Zentrale Voraussetzung für eine Strafbarkeit wegen des genannten Delikts ist das Vorliegen von Daten. Darunter sind auf Datenträgern gespeicherte Daten (vgl. $\$ 74$ Abs. $2 \mathrm{StGB}$ ) zu verstehen, die für den Rechtsverkehr bestimmt sind und den Aussteller erkennen lassen (elektronische Urkunden). ${ }^{36}$ Die elektronisch übermittelte Bestätigung eines negativen COVID-19-Tests erfüllt diese Voraussetzungen. Das Herstellen falscher Daten wird im Gleichklang mit der Urkundenfälschung nach $₫ 223$ StGB so verstanden, dass dabei durch die unbefugte Eingabe neuer Daten der Eindruck erweckt wird, diese stammten von einem anderen als dem tatsächlichen Aussteller. Der Täter täuscht damit über die Identität des Datenaus- 
stellers bzw. der Datenausstellerin. Füllt demnach eine Person ein noch leeres Formular elektronisch aus oder erstellt sie ein neues (elektronisches) Dokument, so werden neue Daten erschaffen und dadurch vorgegeben, die Erklärung (= Daten) stamme vom zuständigen Ministerium bzw. der Republik und eben nicht vom tatsächlichen Aussteller. Verändert eine Person z. B. das Datum des Tests, den Namen der getesteten Person oder das Ergebnis im elektronischen Dokument, so stellt dies eine entsprechende Verfälschung von Daten im Sinne von $\$ 225$ a StGB dar. Damit ist letztlich eine umfassende Strafbarkeit für die in diesem Kapitel angesprochenen Tathandlungen gegeben. Über die Strafpraxis lassen sich derzeit jedoch keine Informationen finden.

\section{Ausgewählte pandemiebedingte Maßnahmen im Strafprozessrecht}

Das Ziel der COVID-19-Maßnahmen, Menschen in ihrem täglichen Lebensbereich vor Ansteckung zu schützen, hat auch zu wesentlichen Veränderungen im Strafverfahrensrecht geführt, da Verfahren zur Aufklärung von Taten sowie der allfälligen Erforderlichkeit von Sanktionen von persönlichen Interaktionen leben. Im Folgenden wird auf die im Rahmen von COVID-19-Maßnahmen erleichterte Möglichkeit von Videovernehmungen sowie die faktische Beschränkung der Öffentlichkeit in der Hauptverhandlung eingegangen, zumal sie derzeit immer noch gelten und auch darüber diskutiert wird, diese infolge der damit verbundenen Vorteile als positive Ergebnisse der Pandemie im Rechtsbestand zu belassen.

Die meisten Änderungen im Bereich des Strafprozessrechts sind durch \9 1. COVID-19-JuBG ${ }^{37}$ ergangen und erfolgten zum Teil unmittelbar in der StPO und ohne Befristung. Diese können in der Zukunft bei vergleichbar gehäuften Erkrankungen leichter nutzbar gemacht werden, indem nicht mehr auf entsprechende Sondergesetze gewartet werden muss. $\$ 9$ 1. COVID-19-JuBG räumt der Bundesministerin für Justiz (BMJ) weitgehende Verordnungsermächtigungen zur Konkretisierung von bestimmten Maßnahmen ein.

37 Vgl. Bundesgesetz betreffend Begleitmaßnahmen zu COVID-19 in der Justiz (1. COVID-19-Justiz-Begleitgesetz - 1. COVID-19-JuBG); BGBl. I 16/2020. Dieses Gesetz wurde zwischenzeitlich mehrmals verlängert und tritt gemäß seinem $\mathbb{} 12$ mit 31. 12. 2021 außer Kraft (vgl. BGBl I 2021/106). 


\subsection{Erleichterte Ermöglichung von Videovernehmungen}

Eine zentrale Änderung betrifft die (erleichterte) Vernehmung von Beschuldigten und Zeugen per Video. Diese Möglichkeit wurde als gleichsame Alternative der „innerstaatlichen Rechtshilfe“ bereits im Jahre 2010 in die Strafprozessordnung $(\mathrm{StPO})^{38}$ eingefügt, ${ }^{39}$ allerdings nur für die U-Haft und begrenzt auf den Fall, dass der Aufenthaltsort eines Zeugen bzw. einer Zeugin oder eines*einer Beschuldigten außerhalb des Sprengels der zuständigen Staatsanwaltschaft (StA) oder des zuständigen Gerichts liegt und eine unmittelbare Vernehmung verfahrensökonomisch nicht zweckmäßiger erscheint. Im Rahmen der COVID-19-Krise wurde diese Möglichkeit nun auf Fälle einer Pandemie ausgedehnt ( $\mathbb{1 7 4}$ Abs. 1 Satz 2 StPO $\left.{ }^{40}\right)$. Videovernehmungen beim Pflichtverhör zur U-Haft-Verhängung wurden damit auch dann zulässig, wenn der*die Beschuldigte direkt in der Justizanstalt des zuständigen Gerichts angehalten wird, um eben COVID-19-Infektionen durch direkten persönlichen Kontakt zwischen Häftlingen, Bediensteten der Justizanstalt und Angehörigen von Gericht und StA möglichst zu verhindern. Mit demselben Ziel wurden Videovernehmungen bei Verhandlungen zur U-Haft-Verlängerung ermöglicht ( $\$ 176$ Abs. $3 \mathrm{StPO}$ ).

Bestand grundsätzliches Verständnis für die Erweiterung der Möglichkeiten einer Videovernehmung im Zusammenhang mit der COVID-19Krise, galt dies weniger für die ebenfalls getroffene Veränderung, Entscheidungen über die Fortdauer der U-Haft ohne Haftverhandlung und damit ohne Vernehmung des*der Gefangenen (zumindest per Video) zu ermöglichen. ${ }^{41}$ Hier scheint der Bequemlichkeitsgedanke eine Rolle gespielt zu haben, denn eine Ansteckung mit COVID-19 kann auch durch eine Videovernehmung verhindert werden und braucht keinen Verhandlungsverzicht. Die Bedenken gegen diese Erweiterung werden noch dadurch gestützt, dass nähere Kriterien für einen Verhandlungsverzicht fehlen. Dem wurde zwar in einer Novellierung von $\$ 9$ Z. 4 1. COVID-19-JuBG durch Ergänzung um den Satz: „soweit im Einzelfall eine Durchführung der Haftverhandlung unter Verwendung technischer Einrichtungen zur Wort-

38 Strafprozeßordnung 1975 (StPO); BGBl. 1975/631.

39 BGBl. I 111/2010.

40 BGBl. I 14/2020.

41 Hierzu räumte $\$ 9$ Z 4 1. COVID-19-JuBG der Bundesministerin für Justiz (BMJ) eine Verordnungsermächtigung ein, von der sie durch BGBl. II 113/2020 Gebrauch gemacht hat. Die entsprechende Bestimmung der Verordnung tritt nach ihrem $₫ 8$ mit 30. 9. 2021 außer Kraft (BGBl. II 281/2021). 
und Bildübertragung nicht möglich ist" ${ }^{\text {42 }}$ versucht, abzuhelfen, eine ausreichende Bestimmtheit wohnt aber auch dieser Neufassung nicht inne. Damit war es für einen Verhandlungsverzicht sowohl ausreichend, dass der*die zuständige Richter*in im Home-Office arbeitete, in freiwilliger Quarantäne war oder über kein leistungsfähiges Internet verfügte, mit dem er*sie eine Videovernehmung durchführen konnte, als auch, dass sich der*die zuständige Richter*in mit der entsprechenden Technik nicht auseinandersetzen wollte und deshalb auf eine Videovernehmung verzichtet hat. Sachgerecht erscheint diese Unbestimmtheit mit Blick auf den Verlust an rechtsstaatlichen Garantien, die eine Vernehmung beinhaltet, nicht.

Videovernehmungen wurden zur Verhinderung von COVID-19-Infektion auch für die Hauptverhandlung ermöglicht. ${ }^{43}$ Nach $\$ 239$ Satz 2 StPO $^{44}$ braucht ein"e verhaftete"r Angeklagt"er nicht mehr zur Hauptverhandlung vorgeführt werden, sondern er"sie kann dieser per Videoschaltung folgen und sich auch per Video einbringen, wodurch seine"ihre Beschuldigtenrechte gewahrt bleiben (sollten). Eine Einschränkung wurde mit Wirkung 1. 6. 2020 dahingehend getroffen, dass im Geschworenenverfahren ein solches Vorgehen nur dann zulässig ist, wenn „es im Einzelfall besonders gewichtige Gründe für unabdingbar erscheinen lassen “ 45 .

Bei allem Verständnis für die Nutzung neuer technischer Möglichkeiten darf bei näherer Betrachtung nicht übersehen werden, dass damit ein beträchtlicher Verlust an Verteidigungsmöglichkeiten einhergehen kann. Dies erscheint insofern wenig bedacht worden zu sein, als sich nirgends eine Regelung findet, wie etwa in Fällen einer Videovernehmung mit der Beiziehung eines Verteidigers bzw. einer Verteidigerin umzugehen ist und wo er*sie sich bei der Vernehmung befinden soll. Naheliegend wäre es, dass der"die Verteidiger"in bei dem"der Beschuldigten in der Haftanstalt sitzt (in entsprechendem Abstand und unter Einhaltung der erforderlichen Sicherheitsbestimmungen), denn eine bloße Zuschaltung des Verteidigers bzw. der Verteidigerin per Video wird vielfach nicht ausreichen, weil auf diese Weise eine unkontrollierte Besprechung zwischen Verteidiger"in und Mandant" in nicht gewährleistet ist. ${ }^{46}$ Für die Hauptverhandlung bedeutet

42 BGBl. I. 24/2020 Art 32 Z 7.

$43 \mathrm{Zu}$ den ebenfalls geschaffenen Möglichkeiten von Videovernehmungen im Rechtsmittelverfahren vgl. Birklbauer in Resch, Corona-HB Kap 16 Rz 51 ff.

44 BGBl. I 14/2020.

$45 \$ 4$ der Verordnung der BMJ zur Verhinderung der Verbreitung von COVID-19; BGB1. II 113/2020 in der Fassung 243/2020.

46 Laut Mitteilung von BMJ Zadić gegenüber dem Parlament wurde auf die Kritik von Rechtsanwält*innen, dass Angeklagte bei Videoverhandlungen nicht immer 
dies freilich, dass die unmittelbare Möglichkeit, sich in die Verhandlung einzubringen, verloren geht, wenn der*die Verteidiger*in nicht unmittelbar vor Ort ist. Dies könnte die Qualität der Verteidigung beeinträchtigen. Insofern wäre es besser, der*die Verteidiger*in würde im Verhandlungssaal sitzen, wodurch aber wiederum eine unumschränkte Kommunikation mit dem Mandanten bzw. der Mandantin nur bei einer Unterbrechung der Hauptverhandlung möglich wäre, im Zuge derer der*die Verteidiger*in den Mandanten bzw. die Mandantin in der Justizanstalt aufsucht. Diese Überlegungen zeigen mit Blick auf den Verhältnismäßigkeitsgrundsatz und Grundrechtsüberlegungen, dass Hauptverhandlungen per Videokonferenz die absolute Ausnahme bleiben müssen und einer Vertagung von Verhandlungen auf Zeiten nach den COVID-19-Beschränkungen der Vorzug zu geben ist, was jedoch mit Blick auf das Beschleunigungsgebot, das für Haftsachen gilt, problematisch sein kann.

In der Praxis gibt es Diskussionen darüber, ob vermehrte Videovernehmungen in Haftsachen nicht ein ökonomischer Weg für die Zukunft sein könnten, vor allem, wenn die Justizanstalt sich nicht in Gerichtsnähe befindet, sondern am Stadtrand und dadurch beträchtliche Ressourcen durch den Transport des*der Gefangenen zur gerichtlichen Vernehmung gebunden werden. Schon die angesprochenen Bedenken zur Kommunikation zwischen Beschuldigtem bzw. Beschuldigter und Verteidiger*in lassen hier jedenfalls Zurückhaltung angebracht erscheinen. Weiters ist die Wahrnehmung von Stimmungen und emotionalen Reaktionen, wie sie etwa für die Beurteilung der Glaubwürdigkeit wesentlich sein können, bei einer Videovernehmung eingeschränkt. Auch dies lässt eine pandemieunabhängige Fortführung von Videovernehmungen in Haftsachen bedenklich erscheinen.

\subsection{Faktische Beschränkung öffentlicher Hauptverhandlungen}

Art. 90 Abs. 1 B-VG ${ }^{47}$ normiert die Verpflichtung zur Öffentlichkeit von Verhandlungen in Strafsachen mit dem Vorbehalt, dass Ausnahmen das Gesetz bestimmt. In ähnlicher Weise kennt Art. 6 Abs. 1 der Europäischen Men-

die Möglichkeit hätten, sich ungestört mit ihrem Verteidiger bzw. ihrer Verteidigerin zu unterhalten, insofern reagiert, als U-Häftlingen ein Handy zur Verfügung zu stellen ist, damit sie sich - ungestört - mit ihren Verteidiger*innen unterhalten können (vgl. Parlamentskorrespondenz Nr. 372 vom 24.04.2020).

Bundes-Verfassungsgesetz, BGBl. 1/1930. 
schenrechtskonvention (EMRK $)^{48}$ den Öffentlichkeitsgrundsatz. Dahinter steht die Überlegung, sowohl eine Kontrolle der Justiz durch das Volk als Ausfluss des Demokratieprinzips zu ermöglichen, als auch den Angeklagten vor einer überbordenden Gerichtsbarkeit zu schützen. ${ }^{49}$

Öffentlichkeit der Hauptverhandlung bedeutet, dass es jedem im Rahmen der technischen Möglichkeiten erlaubt sein muss, einer Verhandlung beizuwohnen. Dies bedeutet freilich nicht, dass der Zutritt zur Hauptverhandlung schlechthin allen interessierten Personen nach ihrem Belieben und ohne Begrenzung ermöglicht werden muss. ${ }^{50}$

In Umsetzung der Verfassungsnormen lässt die StPO Einschränkungen der Öffentlichkeit zu, wobei jedoch die Verkündung des Urteils immer öffentlich erfolgen muss ( $\$ 229$ Abs. 4 StPO). Für die Verhandlung kann die Öffentlichkeit unter anderem wegen Gefährdung der öffentlichen Ordnung oder der nationalen Sicherheit ausgeschlossen werden $(\$ 229$ Abs. 1 Z. 1 StPO). Das Ansteckungsrisiko bei einer Pandemie lässt sich jedoch nicht darunter subsumieren. ${ }^{51}$ Und selbst bei einem Ausschluss der Öffentlichkeit dürfen Richter*in, StA und Strafverteidiger"in, auch wenn sie mit dem konkreten Verfahren nichts zu tun haben, niemals ausgeschlossen werden, sodass das Infektionsrisiko durch einen Ausschluss der Öffentlichkeit allenfalls begrenzt, nicht jedoch völlig unterbunden werden könnte. Dies vor allem auch deswegen, weil Angeklagte, Opfer und Privatbeteiligte verlangen können, dass drei Personen ihres Vertrauens der Zutritt gestattet werde (vgl. $\$ 230$ Abs. 2 StPO). Dadurch soll stets ein Mindestmaß an Kontrolle der Justiz gewährleistet bleiben.

Der Gesetzgeber hat im Zuge der COVID-19-Gesetze letztlich nicht versucht, in den verfassungsrechtlich gewährleisteten Öffentlichkeitsgrundsatz einzugreifen. Selbst wenn verhaftete Angeklagte per Video vernommen wurden (vgl. $\$ 239$ Satz 2 StPO), hatte dies in einem Verhandlungssaal zu geschehen und durften Zuhörer*innen der Hauptverhandlung beiwohnen.

Der Ausschluss der Öffentlichkeit hat durch Beschluss des entscheidenden Gerichts zu erfolgen ( $\$ 229$ Abs. 2 StPO). Nach der Judikatur ist der Öffentlichkeitsgrundsatz aber auch durch die faktische Hinderung Interes-

48 Konvention zum Schutze der Menschenrechte und Grundfreiheiten; BGB1. 210/1958.

49 Vgl. dazu mit Nachweisen etwa Birklbauer, JSt 2009, S. 109; weiters Danek/Mann in WK-StPO $₫ 228 \mathrm{Rz} 4$.

50 Vgl. OGH 15 Os 84/13m = RIS-Justiz RS0128996.

51 Vgl. dazu die Beispiele bei Danek/Mann in WK-StPO $\$ 229 \mathrm{Rz} 2 \mathrm{f}$, welche allgemeine Gesundheitserwägungen nicht zu tragen vermögen. 
sierter, an der Hauptverhandlung teilzunehmen, verletzt. ${ }^{52}$ Dies ist etwa der Fall, wenn das Gericht keine Vorkehrungen trifft, potentiellen Zuhörer*innen nach Versperren des Gerichtsgebäudes den Zutritt zum Verhandlungssaal zu ermöglichen (z. B. durch Hinterlassen einer Telefonnummer). ${ }^{53}$ Insofern wäre ein unzulässiger Ausschluss der Öffentlichkeit etwa vorgelegen, wenn es potentielle Zuhörer*innen zwar bis zum Gericht schafften, aber nicht in das (verschlossene) Gebäude gelangen konnten bzw. die Sicherheitskontrolle eine für sie unüberwindliche Schranke bildete. Auch die Verordnung des Gesundheitsministers, welche infolge von $\$ 2$ COVID-19-Maßnahmengesetz ${ }^{54}$ ergangen war, ${ }^{55}$ konnte in diese Richtung interpretiert werden, denn bei den in $\$ 2$ normierten Ausnahmen vom Verbot des Betretens öffentlicher Orte war die Wahrung von Grundrechten oder die Inanspruchnahme von Grundsätzen der Demokratie, wie sie z. B. hinter dem Öffentlichkeitsgrundsatz stehen, nicht angeführt. Insofern wäre es nach Darstellung seitens der Bundesregierung auch nicht erlaubt gewesen, das Haus zu verlassen, um einer öffentlichen Gerichtsverhandlung als Zuhörer*in beizuwohnen, wodurch der verfassungsrechtliche Öffentlichkeitsgrundsatz in gewisser Weise per Verordnung untergraben war. ${ }^{56}$ Vor diesem Hintergrund war mit Spannung zu erwarten, wie die Judikatur mit COVID-19 bedingten Verletzungen des Öffentlichkeitsgrundsatzes umgehen wird.

Am 26. März 2021 war in Medien die Schlagzeile zu lesen: „OGH: Ausgangssperren mit Öffentlichkeitsgrundsatz vereinbar" ${ }^{57}$ Die dieser Schlagzeile zu Grunde liegende Entscheidung ${ }^{58}$ ging auf die Verletzung des Öffentlichkeitsgrundsatzes letztlich nicht ein, sondern stellte schlicht fest, dass im Zeitpunkt der Hauptverhandlung die gesetzgeberischen Maßnahmen zur Verhinderung der Verbreitung von COVID 19 für Strafsachen anders als etwa für bürgerliche Rechtssachen - keine Beschränkungen für die Durchführung von Hauptverhandlungen vorsahen. Darüber hinaus habe der Verteidiger im Rechtsmittel auch keinen faktischen Ausschluss der

52 Vgl. OGH 15 Os 61/02 = RIS-Justiz RS0117048.

53 Vgl. OGH 13 Os 102/11s.

54 Vgl. BGBl. I 12/2020.

55 Vgl. BGBl. II 98/2020 in der Fassung 162/2020. Diese Verordnung ist entsprechend ihrem $\$ 7$ mit 30. 4. 2020 außer Kraft getreten.

56 Kritisch zu dieser Maßnahme vgl. Birklbauer, JSt 2020, S. 197.

57 ORF: OGH: Ausgangssperren mit Öffentlichkeitsgrundsatz vereinbar, in: https:// orf.at/stories/3206831/ [1.7.2021].

58 Vgl. OGH 14 Os 6/21w = RIS-Justiz RS0133515 = JSt 2021, S.308 mit Anm. Birklbauer. 
Öffentlichkeit - etwa durch versperrte Gerichtsgebäude - geltend gemacht. Zur Frage, ob die Ausgangssperren mit dem Öffentlichkeitsgrundsatz vereinbar waren, hat sich der OGH letztlich nicht geäußert, weil es kein rechtliches Hindernis für potentiell interessierte Zuhörer*innen gab, an einer Hauptverhandlung teilzunehmen.

\section{Ausgewählte pandemiebedingte Maßnahmen im Bereich des Strafvollzugs}

Das bereits erwähnte 1. COVID-19-JuBG hat der BMJ auch im Bereich des Strafvollzugs verschiedene Verordnungsermächtigungen eingeräumt. Durch den Verweis auf die sinngemäße Anwendung des I. Hauptstücks des genannten Gesetzes stehen diese unter dem Leitgedanken, „einerseits die Gefahr eines Übergreifens des Virus auf den Strafvollzug möglichst hintanzuhalten, andererseits aber auch das System des Strafvollzugs, einschließlich seiner rechtsstaatlichen Garantien, möglichst aufrechtzuerhalten bzw. zu bewahren, um so bestmöglich zur Sicherheit der Allgemeinheit, aber nicht zuletzt auch der Strafvollzugsbediensteten und der im Strafvollzug befindlichen Personen beizutragen ".59 Die folgenden Ausführungen betreffen beispielshaft die Möglichkeiten zum Haftaufschub sowie die Einschränkungen des Besuchsverkehrs gegenüber Gefangenen. ${ }^{60}$

\subsection{Möglichkeiten zum Haftaufschub}

\$10 Z. 1 1. COVID-19-JuBG erlaubt es der BMJ, per Verordnung das Unterbleiben der Aufforderung zum Strafantritt für eine*n auf freiem Fuß befindlichen rechtskräftig zu einer Freiheitsstrafe Verurteilten ( $\$ 3$ Abs. 2 erster Satz StVG ${ }^{61}$ ) für die Dauer von COVID-19-Maßnahmen, nach derzeitigem Stand also längstens bis 31. 12. 2021, anzuordnen, während $\$ 10$ Z. 4 1. COVID-19-JuBG generell einen Vollzugsaufschub ( $\mathbb{6} 6 \mathrm{Abs.} 1 \mathrm{StVG}$ ) für Freiheitsstrafen bis zu drei Jahren ermöglicht, um den Zugang in den Strafvollzug zu beschränken. Darüber hinaus ermächtigt $\mathbb{1} 10 \mathrm{Z} .31$. COVID-19-JuBG dazu, mit COVID-19 infizierte Personen oder solche, die we-

59 Initiativantrag (IA) Nr. 397/A 27. GP S. 39.

$60 \mathrm{Zu}$ den anderen Änderungen im Bereich des Strafvollzugs vgl. Birklbauer in Resch, Corona-HB Kap 16 Rz 79 ff.

61 Bundesgesetz vom 26. März 1969 über den Vollzug der Freiheitsstrafen und der mit Freiheitsentziehung verbundenen vorbeugenden Maßnahmen (Strafvollzugsgesetz - StVG); BGBl. 144/1969. 
gen Kontakts mit infizierten Personen unter Quarantäne stehen, gemäß $\$ 5$ StVG als (ursprünglich) und $\mathbb{\$} 133$ StVG als (nachträglich) vollzugsuntauglich einzustufen. Dies bedeutet nicht, dass diese Personen in jedem Fall auf freiem Fuß bleiben oder enthaftet werden, sondern allenfalls eine Haft anderer Art (z. B. in einer öffentlichen Krankenanstalt) vollzogen werden kann, wenn die Kapazitäten der Justizanstalten zur medizinischen Behandlung und zu besonderen Quarantänemaßnahmen erschöpft sind. ${ }^{62}$ Eine solche Haft anderer Art in einer Krankenanstalt ist für den Verurteilten günstig, weil er durch seine Krankheit ohnehin in seiner Freiheit beschränkt ist und mit dem „besonderen Strafvollzug“ im Ergebnis einen Teil seiner Freiheitsstrafe auf diese Weise verbüßt.

Die Umsetzung dieser positiv zu bewertenden gesetzlichen Ermächtigungen durch die BMJ war bemerkenswert. Nach $\$ 2$ der entsprechenden Verordnung ${ }^{63}$ war zwar bei einem"einer Verurteilten, der*die sich auf freiem Fuß befand, ein Strafantritt aufzuschieben ${ }^{64}$, davon wurden aber Verurteilte wegen einer in $\$ 33$ Abs 2 StGB umschriebenen Tat (im Wesentlichen Gewalt im sozialen Nahbereich, in hohem Ausmaß oder unter Waf-

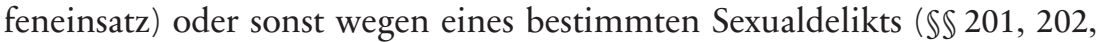
205, 205a, 206, 207, 207a oder 207b StGB) ausgenommen, obwohl ihre Gefährlichkeit als so gering eingestuft wurde, dass sie nicht in U-Haft waren. Nach dem Terroranschlag in Wien im November 2020 wurde diese Bestimmung um Terrordelikte ( $\mathbb{S} 2278 \mathrm{~b}$ bis $278 \mathrm{~g}$ und $\$ 282 \mathrm{a}$ StGB) ergänzt. ${ }^{65}$ Durch diesen Ausschluss bestimmter Tätergruppen vom Vollzugsaufschub war die Verordnung deutlich enger als es das Gesetz erforderte. Damit wurde letztlich ausgedrückt, dass solche Straftäter*innen weniger vor COVID-19Infektionen zu schützen sind, was unter Verhältnismäßigkeitsgesichtspunkten und mit Blick auf die Menschenwürde mehr als bedenklich ist.

62 Vgl. IA 397/A 27. GP S. 39.

63 Vgl. Verordnung der BMJ über besondere Vorkehrungen im Anwendungsbereich des StVG zur Verhinderung der Verbreitung von COVID-19 vom 26. 3. 2020; BGBl. II 120/2020. Die Verordnung tritt nach ihrem derzeit geltenden $\$ 10 \mathrm{mit}$ 30. 9. 2021 außer Kraft (BGBl. II 2021/282).

64 Das ursprüngliche Aufschubdatum bis 30. 4. 2020 wurde mittlerweile in bestimmten Fällen sogar auf 30. 6. 2021 geändert (BGBl. II 2021/125). Obwohl die Verordnung nach derzeitigem Stand noch bis 30.9.2021 gilt, hat sie in diesem Punkt ab 1.7.2021 keinen Anwendungsbereich mehr. Vgl. BGBl. II 2020/493. 


\subsection{Besuchsbeschränkungen}

Menschen in Haft sind in ihren Kontakten zur Außenwelt von vornherein stark beschränkt. Um den vorhandenen persönlichen und familiären Beziehungen überhaupt eine Chance zu geben, sind sie - auch mit Blick auf die Resozialisierung - aber entscheidend. Da durch Besuche ein Infektionsrisiko besteht, war die Einschränkung der Besuchsmöglichkeiten im Rahmen der COVID-19-Krise naheliegend. Vor diesem Hintergrund ermächtigte $₫ 10$ Z 5 1. COVID-19-JuBG die BMJ, den Besuchsverkehr $(\mathbb{S} 93$ StVG) für die Dauer der vorläufigen COVID-19-Maßnahmen auf telefonische Kontakte zu beschränken oder sonstige Beschränkungen des Verkehrs mit $\operatorname{der} A u$ ßenwelt vorzusehen, wobei sich für diese Beschränkungen keine weitere Determinierung fand. ${ }^{66}$ Mit $\$ 5$ der entsprechenden Verordnung ${ }^{67}$ wurde der Besuchsverkehr ( $\$ 93 \mathrm{StVG}$ ), mit Ausnahme der Besuche von Vertreter*innen öffentlicher Stellen und von Betreuungseinrichtungen sowie von Rechtsbeiständen ( $\mathbb{9} 96 \mathrm{StVG}$ ) letztlich bis zum 10. 5. $2020^{68}$ auf telefonische Kontakte beschränkt. Das Besuchsverbot zwischen 1. und 10. 5. 2020 wurde insofern gelockert, als ab diesem Zeitpunkt Besuche einzelner Personen zugelassen wurden, bei unter 14-jährigen Besucher*innen auch mit einer erwachsenen Begleitperson. Damit sollte einerseits gewährleistet werden, dass Infektionen von Strafgefangenen und Vollzugspersonal mit COVID-19 nach Möglichkeit verhindert werden, andererseits doch auch den Rechten von Gefangenen entsprochen werden. Insgesamt kann diese Regelung als zunächst verhältnismäßig eingestuft werden.

Die Verschärfung der Pandemie führte auch zu neuen Besuchsbeschränkungen im Strafvollzug. So wurde $₫ 5$ der genannten Verordnung mit Wirkung vom 21. 11. 2020 wieder eingeführt und zunächst mit 13. 12. 2020 befristet. ${ }^{69}$ In den darauffolgenden Tagen galt sie infolge der Lockerungen rund um Weihnachten nicht. Mit Wirkung 30. 12. 2020 wurde sie erneut

66 Eine inhaltsgleiche Ermächtigung sieht $\$ 9$ Z. 5 1. COVID-19-JuBG für den Bereich der U-Haft ( $\$ 188$ Abs 1 StPO) vor.

67 Vgl. BGBl. II 120/2020.

68 Vgl. BGBl. II 184/2020. Zur inhaltsgleichen Regelung für die U-Haft vgl. $₫ 5$ der Verordnung der BMJ, mit der zur Verhinderung der Verbreitung von COVID-19 besondere Vorkehrungen in Strafsachen getroffen werden (BGBl. II 113/2020 in der Fassung BGBl. II 180/2020).

69 Vgl. BGBl II 2020/493. 
eingeführt ${ }^{70}$ und letztlich bis 2. 5. 2021 verlängert. ${ }^{71}$ Dass nicht mehr als zwei Besucher*innen gleichzeitig zum Besuch eines*einer Strafgefangenen zugelassen werden, gilt nach derzeitigem Stand noch bis zum 30. 9. 2021. Die Tendenz, die Grundrechte von Personen, die keine Lobby haben, über Gebühr stark einzuschränken, zeigt sich auch in diesem Bereich. ${ }^{72}$

\section{Zusammenfassende Schlussfolgerungen}

Im Bereich des materiellen Strafrechts gab es bei den Delikten zum Schutz von Leib und Leben ( $\mathbb{S} 75 \mathrm{ff} S \mathrm{tGB}$ ) sowie jenen zum Schutz der „Volksgesundheit“" ( $\$ \$ 178 \mathrm{f} \mathrm{StGB)} \mathrm{keine} \mathrm{pandemiebedingten} \mathrm{Veränderungen.} \mathrm{Die}$ Rechtspraxis, die vor allen hinsichtlich der abstrakten Gefährdungsdelikte zum Schutz der allgemeinen Gesundheit ( $\$ \mathbb{S} 178 \mathrm{f}$ StGB) zunächst dem (gesundheitspolitischen) Mainstream folgte und die Missachtung eines Absonderungsbescheids als ausreichend für eine Strafbarkeit ansah, ist mittlerweile einer differenzierten Ansicht gewichen, nach der es auf bestimmte Parameter ankommt, damit eine Person als ausreichend infektiös gelten kann, um gesundheitsgefährdend zu sein.

Mit den Impfmöglichkeiten ist das Phänomen des „Vordrängens“ in Erscheinung getreten, mit den zunehmenden Lockerungen, für die negative Testergebnisse Voraussetzungen waren, gingen Fälschungen von Testbestätigungen einher, die sich beim so genannten "Grünen Pass“ wohl fortsetzen werden. Das Vordrängen bei Impfungen ist ohne strafrechtliche Relevanz, sofern die vorgesehene Reihenfolge nicht als Ausgleich für eine „Spende“ oder einen sonstigen Vorteil durchbrochen wird. Für Fälschungen von Testbestätigungen können die Delikte der Urkundenfälschung $(\mathbb{2} 223 \mathrm{StGB})$ bzw. Datenfälschung $(\$ 225 \mathrm{a}$ StGB$)$ einschlägig sein und damit ein vielfach größeres strafrechtliches Risiko begründen als das Vordrängen bei den Impfungen, was unter rechts- und kriminalpolitischen Gesichtspunkten diskussionswürdig ist.

Im Bereich des Strafverfahrens wurde die Diskussion, ob der Ausschluss der Öffentlichkeit von Hauptverhandlungen durch pandemiebedingte

70 Vgl. BGBl II 2020/624.

71 Vgl. BGBl II 2021/183, wobei infolge unterschiedlicher regionaler Entwicklungen die Beschränkung am Ende nur mehr für die Bundesländer Wien und Niederösterreich gegolten haben.

72 Kritisch zu diesen Beschränkungen und ihre Ausgestaltung mit Blick auf die Grundrechte von Strafgefangenen und ihren Angehörigen vgl. Czech, JSt 2021, S. 271. 
Ausgangsbeschränkungen grundrechtswidrig war, letztlich vom OGH dahingehend entschieden, dass es kein rechtliches Hindernis für potentiell interessierte Zuhörer"innen gab, an einer Hauptverhandlung teilzunehmen, und faktische Hindernisse im Einzelfall nicht geltend gemacht wurden. Dies erweckt ein bisschen den Eindruck: Es war ja nicht so schlimm!

Beschuldigtenvernehmungen per Video zur Eindämmung von COVID-19 wurden im Strafverfahren zum Teil ein fester Bestandteil im Verfahren, der von manchen positiv bewertet wurde im dem Sinne: es hat alles überraschend gut funktioniert. Insofern verwundert es nicht, wenn derzeit sogar die Diskussion dahingehend geführt wird, diese Möglichkeit pandemieunabhängig bestehen zu lassen. Dass Grundrechtsaspekte weitgehend dagegensprechen, wurde dargelegt.

Ein Rückblick auf eineinhalb Jahre Pandemie führt zusammenfassend zum Resümee, dass das Strafrecht kein taugliches Mittel zum Krisenmanagement war (und ist). Insofern verdient der vom Gesetzgeber gewählte maßvolle Einsatz des Strafrechts mit Blick auf den Ultima-Ratio-Grundsatz Zustimmung. Lob gebührt auch der Justiz, die mit Fortdauer der Pandemie den politischen ,Mainstream' kritisch hinterfragte und eine Missachtung des Absonderungsbescheides nicht mehr für die Gefährdung der ,Volksgesundheit" und damit eine Strafbarkeit wegen $\$ \mathbb{S} 178 \mathrm{f} \mathrm{StGB}$ ausreichen ließ. Wenn es im Bereich des Strafverfahrens nun jedoch darum geht, das ,Positive' von Social Distancing bei Verhandlungen unabhängig von der Pandemie für die Zukunft mitzunehmen, sind jedenfalls mit Blick auf Videovernehmungen Bedenken angebracht. Zur Feststellung der Schuld und zur Festsetzung einer strafrechtlichen Sanktion bedarf es direkter und unmittelbarer Kommunikation, um auch ,Botschaften zwischen den Zeilen` zu erfassen, die in Videokommunikationen schlicht unterzugehen drohen.

\section{Literatur:}

Birklbauer, Alois: Die Öffentlichkeit der Hauptverhandlung. Ein Prozessgrundsatz im Spannungsfeld zwischen Beschuldigten-, Opfer- und Bevölkerungsinteresse, in: Journal für Strafrecht (JSt) 7/4 (2009) 109-117.

Birklbauer, Alois: Kapitel 16: Strafrecht, in: Resch, Reinhard (Hg): Das CoronaHandbuch. Österreichs Rechtspraxis zur aktuellen Lage, Wien: Manz 2020, 403432 (= Corona-HB Kap $16 \mathrm{Rz}$ xx).

Birklbauer, Alois: Die Auswirkung der COVID-19-Gesetze auf das Strafrecht, in: Journal für Strafrecht (JSt) 7/3 (2020) 197-203. 
Birklbauer, Alois: Strafrechtliche Haftung der Gesundheitsberufe, in: Resch, Reinhard/Wallner, Felix (Hg): Handbuch Medizinrecht. 3. Auflage, Wien: LexisNexis 2020 (= Kap X Rz xx).

Burgstaller, Manfred/Fabrizy, Ernst Eugen: $₫ 83$ StGB, in: Höpfel, Frank/Ratz, Eckart (Hg): Wiener Kommentar zum Strafgesetzbuch. 2. Auflage, Wien: Manz 2018 (= WK ${ }^{2}$ StGB).

Czech, Peter: Besuchsbeschränkungen in Justizanstalten zur Bekämpfung von Covid-19, in: Journal für Strafrecht (JSt) 8/3 (2021) 271-278.

Danek, Michael/Mann, Irene: $\$ 228$ StPO, in: Fuchs, Helmut/Ratz, Eckart (Hg): Wiener Kommentar zur Strafprozessordnung, Wien: Manz 2017 (= WK-StPO).

Leukauf, Otto/Steininger, Herbert/Tipold, Alexander: $\mathbb{S} 178$ Strafgesetzbuch. Kommentar. 4. Auflage, Wien: Linde 2017.

Murschetz, Verena: $\$ \$ 178,179$ StGB, in: Höpfel, Frank/Ratz, Eckart (Hg): Wiener Kommentar zum Strafgesetzbuch. 2. Auflage, Wien: Manz 2020 (= WK ${ }^{2}$ StGB).

Neuhofer, Magdalena: Strafrechtliche Aspekte der Fälschung von Antigen-Schnelltestergebnissen, in: Journal für Strafrecht (JSt) 8/3 (2021) 231-238. 